\section{Characteristics associated with media use in early adolescence}

\author{
Características associadas com o uso da mídia \\ no início da adolescência
}

\section{Características asociadas con el uso de medios de comunicación en la adolescencia temprana}

\begin{abstract}
This study aimed to identify socioeconomic and behavioral characteristics associated with media use in early adolescence. The sample was comprised of 1,680 adolescents (884 girls and 796 boys) aged 13, attending private and public schools in Porto, Portugal, during 2003/2004. Adolescents completed questionnaires pertaining to demographic, social and behavioral characteristics, including the time spent watching television and playing computer games on week and weekend days. Logistic and proportional ordinal regressions showed that attending public schools, sleeping less time, using tobacco and presenting low levels of physical activity were factors associated with high media use. In boys living with one of their parents as well as living with younger and older parents were also associated with high media use. Besides the association with less healthier behaviours, we also found higher media use among adolescents from lower social classes and less structured families, which may increase their exposure to negative influence of the media.
\end{abstract}

Communications Media; Adolescent; Adolescent Behavior; Television
José Godinho 1,2 Joana Araújo 1,2 Henrique Barros 1,2 Elisabete Ramos 1,2

\section{Resumo}

O objetivo do estudo foi identificar as características socioeconômicas e comportamentais associadas ao uso da mídia, no início da adolescência. A amostra foi constituída por 1.680 adolescentes (884 garotas e 796 garotos) de 13 anos que frequentavam escolas públicas $e$ privadas no Porto, Portugal, em 2003/2004. Eles preencheram questionários com informação sobre características sociodemográficas e comportamentais, incluindo tempo gasto com o uso da televisão e computador, em dias da semana e fins de semana. Regressões logísticas ordinais e proporcionais mostraram que os adolescentes das escolas públicas, os que dormiam menos, consumiam tabaco e praticavam pouca atividade física tinham maior propensão a usar a mídia mais tempo. Garotos que viviam com um dos pais ou que tinham pais mais jovens ou mais velhos usavam mais tempo a mídia. Assim, além da associação com comportamentos menos saudáveis, constatou-se um maior uso da mídia nos adolescentes de classe social inferior e de famílias menos estruturadas, o que pode aumentar a sua permeabilidade à influência negativa da mídia.

Meios de Comunicação; Adolescente; Comportamento do Adolescente; Televisão 


\section{Introduction}

The role of exposure to media had been studied since the middle of the 20th century, when television exposure became frequent 1 . Given earlier and massive dissemination of television and the emergence of concerns about its impact, much of the existing research about media exposure focuses primarily on this media 2 . In line with the fast advancement in technologies, more recently newer forms of media became ubiquitous, including personal computers and video games, as well as the internet 1 . However, these remain less studied than television ${ }^{3}$.

Television and computer exposure have potential benefits and risks 4,5. Studies with children showed a tendency to imitate positive social behaviours, such as altruism and helping 6 . Computer exposure can also supply increased learning opportunities, fast access to high amounts of information, as well as training of fine motor and visual skills 7. On the other hand, high quantitative and negative qualitative media exposure may result in displacement of protective activities on the intellectual, physical and social levels, such as practicing physical activities or having real social interactions that are fundamental to a harmonious growing mind and intellect 4,8,9,10,11,12,13. Additionally, exposure to poor models can also promote inadequate behaviours 6 . Higher media use was associated with higher risk of physical damage, namely, muscoeskeletal and visual disturbances and irregular sleep patterns 4,5,7,14,15. Psychological consequences, including depression, aggressive behaviours and addictive use 4,5,7 and social isolation 5 were also described. These effects were particularly relevant in adolescence since during this period of life adolescents become more vulnerable to the influences of their external milieu, where peers and media represent important elements 16 .

Additionally, adolescence is a critical period during which the acquisition of autonomy is expected and parental control declines, allowing for an increase in the time available for adolescents to self-manage their activities, including exposure to television and computers 2. Given the negative impact of excessive exposure to media 4,5,7,14,15, especially at early adolescence, it is fundamental to improve knowledge about the characteristics associated with higher use to allow a better design and implementation of strategies oriented to reduce this exposure and subsequent prejudicial impact. Therefore, the aim of this study was to identify socioeconomic and behavioural characteristics associated with media use, in a sample of 13-year-old adoles- cents, in order to provide additional evidence that may help to optimize and target relevant interventions.

\section{Methods}

\section{Participants}

The study was developed as part of the Epidemiological Health Investigation of Teenagers in Porto (EPITeen). Eligible participants were all adolescents born in 1990 and enrolled at public and private schools in Porto during the 2003/2004 school year 17. The executive boards of the 51 schools in Porto were contacted in order to include all 13-year-old adolescents and their families (since teaching is compulsory in Portugal until the age of 15, school is the best sample frame for a population-based approach). Both adolescents and respective parents received a written explanation about the aim and design of the study. This information was also clarified during meetings held with adolescents and their families, in each school. In brief, we identified 2,787 adolescents born in 1990, of whom $2,160$ (1,651 at public and 509 at private schools $)$ agreed to participate and provided information for at least part of the planned assessment. This resulted in $77.5 \%$ overall participation, with $77.7 \%$ participation at public and $77 \%$ at private schools, $p=0.709$. The study was approved by the ethics committee of the São João University Hospital. Written informed consent was achieved both from the adolescents and their legal guardians, and data confidentiality and protection was assured through the creation of policies and procedures.

For this analysis, 247 adolescents, whose parents did not return the questionnaires, were excluded. Moreover, 233 adolescents were also excluded because they had missing or inconsistent data (for example, when reported media use was greater than 24 hours per day) regarding media. The final sample included 1,680 adolescents, 884 girls and 796 boys.

Those excluded were significantly more male and more frequently attended public schools, had repeated school years, scored $\geq 13$ in the depressive symptoms scale and had the youngest and least educated parents. No significant differences were found regarding practice of physical exercise, smoking, body mass index, sleep time and presence of any chronic illness. 


\section{Data collection}

The evaluation included two self-administered questionnaires (one completed at home, another at school) and a physical examination.

The home questionnaire, answered by adolescents and parents, inquired about the demographic, social, behavioural and clinical history of the adolescent and the family. As part of the home questionnaire, the amount of time spent watching television and using a computer was evaluated separately for each one and separately for week and weekend days. Based on this information, and for each media, we computed the daily average considering the sum of the five week days and the two weekend days, divided by seven days. Total media use per day was calculated as the sum of the daily time using the television and computer.

Sleep duration was estimated by the difference between self-reported usual bedtimes and wake-up times.

Parental education level was measured as the number of successfully completed years of formal schooling and in the analysis we used the information from the parent with the higher education level. Each school was considered public if it was state administered, or private, when it was not administered by local or national governments. Parental education level and the type of school were used as indicators of socio-economic class. We also gathered information about who adolescents lived with. Due to the small number of adolescents living with only one parent or living in another situation, we classified the adolescents in two categories, according to living with both parents: yes or no.

At school, during the research team visit, adolescents answered an additional questionnaire comprising further information on current year school frequency, physical activity, smoking, drinking and depressive symptoms. A repeated school year was considered to have occurred when the adolescent was found not to be in the school year expected for their age. For tobacco use, adolescents were classified as non-smokers if they had never smoked; and as ever smokers if they had tried smoking or if they smoke, irrespectively of the frequency and quantity. We also assessed the frequency of self-reported physical activity, and adolescents were categorized into two categories (less than once a week and once or more a week). As part of this questionnaire, the depressive symptomatology was evaluated through the Second Edition of Beck Depression Inventory (BDI-II) 18 , previously validated in Portuguese adolescents, with an established cut-off of 13 points to categorize non-depressed and depressed adolescents 19 .

During the school visit, anthropometrics were obtained with the subject in light indoor clothes and no shoes. Weight was measured in kilograms, to the nearest tenth, using a digital scale (Tanita TBF-300, Tanita Corporation of America Inc., Arlington Heights, USA), and height was measured (in centimetres, to the nearest tenth) using a portable stadiometer. Body mass index (BMI) was calculated and classified according to the age- and sex-specific BMI reference percentiles developed by the United States Centers for Disease Control and Prevention 20. Participants were considered overweight if BMI was equal to or greater than the 85th percentile.

\section{Statistical analysis}

Data were analysed separately for boys and girls. Proportions were compared using the chi-square test or Fisher's exact test. To estimate the magnitude of the association between the adolescents' or parents' characteristics and television (categorized into $\leq 1$ hour; $>1 \& \leq 2$ hours $\&>2$ hours) or total media exposure (categorized into $\leq 2$ hours; $>2 \& \leq 3$ hours $\&>3$ hours), proportional odds ratio and $95 \%$ confidence intervals (\%95CI) were calculated using ordinal regression. Regarding computer utilization (divided into two categories, $\leq 2$ hours $\&>2$ hours), we calculated the odds ratio (OR) and 95\%CI, using unconditional logistic regression. All models were adjusted for parental education.

Statistical analyses were performed using the SPSS, version 17.0 (IBM Corp., Armonk, USA).

\section{Results}

Considering the daily average of television, the prevalence of adolescents watching more than 2 hours per day was $48.5 \%$ in girls and $48 \%$ in boys. Considering the total time of exposure (both television and computer), $62.7 \%$ of girls and $76.9 \%$ of boys reported a use that was greater than 2 hours per day. No significant gender differences were found regarding the time spent watching television. However, boys spend significantly more time using the computer than girls, especially at the weekend (Table 1).

Girls that had parents with lower educational levels, who were from a public school, had repeated a school year and practiced exercise less frequently, reported significantly more time watching television, which was reflected in differences in the total time of media exposure. With regard to depressive symptoms, greater 
Adolescents' television, computer and total media use, by gender.

\begin{tabular}{|c|c|c|c|}
\hline & $\begin{array}{l}\text { Girls } \\
\text { n (\%) }\end{array}$ & $\begin{array}{l}\text { Boys } \\
n(\%)\end{array}$ & p-value \\
\hline \multicolumn{4}{|l|}{ Television use } \\
\hline Week days (hours/day) & & & 0.499 \\
\hline$\leq 1$ & $268(30.3)$ & $263(33.0)$ & \\
\hline$>1 \leq 2$ & $297(33.6)$ & $247(31.0)$ & \\
\hline$>2 \leq 3$ & $140(15.8)$ & $134(16.8)$ & \\
\hline$>3$ & $179(20.2)$ & $152(19.1)$ & \\
\hline \multicolumn{4}{|l|}{ Weekend (hours/day) } \\
\hline$\leq 1$ & $68(7.7)$ & $58(7.3)$ & 0.712 \\
\hline$>1 \leq 2$ & $111(12.5)$ & $114(14.3)$ & \\
\hline$>2 \leq 3$ & $142(16.1)$ & $132(16.6)$ & \\
\hline$>3$ & $563(63.7)$ & $492(61.8)$ & \\
\hline \multicolumn{4}{|l|}{ Global daily average (hours/day) } \\
\hline$\leq 1$ & $141(16.0)$ & $134(16.8)$ & 0.968 \\
\hline$>1 \leq 2$ & $314(35.5)$ & $280(35.2)$ & \\
\hline$>2 \leq 3$ & $216(24.4)$ & $194(24.4)$ & \\
\hline$>3$ & $213(24.1)$ & $188(23.6)$ & \\
\hline \multicolumn{4}{|l|}{ Computer use } \\
\hline Weekdays (hours/day) & & & $<0.001$ \\
\hline$\leq 1$ & $754(85.3)$ & $548(68.8)$ & \\
\hline$>1 \leq 2$ & $81(9.2)$ & $143(18.0)$ & \\
\hline$>2 \leq 3$ & $31(3.5)$ & $47(5.9)$ & \\
\hline$>3$ & $18(2.0)$ & $58(7.3)$ & \\
\hline Weekend (hours/day) & & & $<0.001$ \\
\hline$\leq 1$ & $620(70.1)$ & $208(26.1)$ & \\
\hline$>1 \leq 2$ & $137(15.5)$ & $194(24.4)$ & \\
\hline$>2 \leq 3$ & $51(5.8)$ & $125(15.7)$ & \\
\hline$>3$ & $76(8.6)$ & $269(33.8)$ & \\
\hline Global daily average (hours/day) & & & $<0.001$ \\
\hline$\leq 1$ & $724(81.9)$ & $418(52.5)$ & \\
\hline$>1 \leq 2$ & $107(12.1)$ & $240(30.2)$ & \\
\hline$>2 \leq 3$ & $31(3.5)$ & $73(9.2)$ & \\
\hline$>3$ & $22(2.5)$ & $65(8.2)$ & \\
\hline $\begin{array}{l}\text { Global daily average of total media use (television and } \\
\text { computer) (hours/day) }\end{array}$ & & & $<0.001$ \\
\hline$\leq 1$ & $85(9.6)$ & $21(2.6)$ & \\
\hline$>1 \leq 2$ & $245(27.7)$ & $163(20.5)$ & \\
\hline$>2 \leq 3$ & $210(23.8)$ & $210(26.4)$ & \\
\hline$>3$ & $344(38.9)$ & $402(50.5)$ & \\
\hline
\end{tabular}

computer use was found among girls that scored $\geq 13$ on the BDI-II (Table 2 ).

Boys that lived with only one of their parents, whose parents were younger and less educated, and that attended public schools watched television significantly more time and played more frequently on the computer. Additionally, boys that repeated the school year and those that reported lower sleep duration spent more time watching television, reproducing similar results on the total media use (Table 3).

In females, concerning the likelihood of change, girls attending private schools had lower odds of being exposed to television for longer (proportional OR $=0.59$; 95\%CI: $0.43-0.81$ ), as well as those who practiced exercise more than 
Daily mean of television, computer and total media use, according to parents' and adolescents' characteristics, in girls.

\begin{tabular}{|c|c|c|c|c|c|c|c|c|c|c|c|c|c|}
\hline & \multicolumn{5}{|c|}{ Television (hours/day) } & \multicolumn{4}{|c|}{ Computer games (hours/day) } & \multicolumn{4}{|c|}{ Total media (hours/day) } \\
\hline & $\begin{array}{c}\leq 1 \\
n(\%)\end{array}$ & $\begin{array}{c}>1 \leq 2 \\
n(\%)\end{array}$ & $\begin{array}{c}>2 \leq 3 \\
n(\%)\end{array}$ & $\begin{array}{c}>3 \\
\mathrm{n}(\%)\end{array}$ & $\mathrm{p}$-value & $\begin{array}{c}\leq 1 \\
n(\%)\end{array}$ & $\begin{array}{c}>1 \leq 2 \\
n(\%)\end{array}$ & $\begin{array}{c}>2 \\
n(\%)\end{array}$ & $\mathrm{p}$-value & $\begin{array}{c}\leq 2 \\
n(\%)\end{array}$ & $\begin{array}{c}>2 \leq 3 \\
n(\%)\end{array}$ & $\begin{array}{c}>3 \\
\mathrm{n}(\%)\end{array}$ & $\mathrm{p}$-value \\
\hline \multicolumn{14}{|c|}{$\begin{array}{l}\text { Living with both } \\
\text { parents }\end{array}$} \\
\hline No & $26(12.8)$ & $72(35.5)$ & $50(24.6)$ & $55(27.1)$ & 0.495 & $173(85.2)$ & $19(9.4)$ & $11(5.4)$ & 0.424 & $36(17.7)$ & 79 (38.9) & $88(43.3)$ & 0.222 \\
\hline Yes & $104(16.3)$ & $232(36.3)$ & $157(24.6)$ & $146(22.8)$ & & $521(81.5)$ & $81(12.7)$ & $37(5.8)$ & & $140(21.9)$ & $262(41.0)$ & $237(37.1)$ & \\
\hline \multicolumn{14}{|l|}{$\begin{array}{l}\text { Parents' age } \\
\text { (years) }\end{array}$} \\
\hline$\leq 40$ & $17(13.0)$ & $46(35.1)$ & $30(22.9)$ & $38(29.0)$ & 0.633 & $103(78.6)$ & $18(13.7)$ & $10(7.6)$ & 0.525 & $19(14.5)$ & $51(38.9)$ & $61(46.6)$ & 0.162 \\
\hline $41-50$ & $65(15.5)$ & $158(37.7)$ & $97(23.2)$ & $99(23.6)$ & & $348(83.1)$ & $47(11.2)$ & $24(5.7)$ & & $100(23.9)$ & $158(37.7)$ & $161(38.4)$ & \\
\hline$\geq 51$ & $29(17.2)$ & $52(30.8)$ & $45(26.6)$ & $43(25.4)$ & & $146(86.4)$ & $15(8.9)$ & $8(4.7)$ & & $33(19.5)$ & $70(41.4)$ & $66(39.1)$ & \\
\hline \multicolumn{14}{|c|}{$\begin{array}{l}\text { Parental } \\
\text { education (years) }\end{array}$} \\
\hline$\leq 6$ & $26(10.3)$ & $83(32.9)$ & $62(24.6)$ & $81(32.1)$ & $<0.001$ & 228 & $10(4.0)$ & $9(3.6)$ & 0.485 & $34(14.4)$ & $96(40.7)$ & $106(44.9)$ & $<0.001$ \\
\hline $7-12$ & $59(14.2)$ & $148(35.6)$ & $104(25.0)$ & $105(25.2)$ & & $388(94.4)$ & $14(3.4)$ & $9(2.2)$ & & 73 (18.3) & $161(40.4)$ & 165 (41.4) & \\
\hline$>12$ & $55(24.2)$ & 90 (39.6) & $48(21.1)$ & $34(15.0)$ & & $212(95.9)$ & $6(2.7)$ & $3(1.4)$ & & $74(34.1)$ & $82(37.8)$ & $61(28.1)$ & \\
\hline \multicolumn{14}{|c|}{ Type of school } \\
\hline Public & 80 (12.5) & $216(33.7)$ & $171(26.7)$ & $174(27.1)$ & $<0.001$ & $511(79.7)$ & 85 (13.3) & $45(7.0)$ & 0.018 & $97(15.1)$ & $266(41.5)$ & 278 (43.4) & $<0.001$ \\
\hline Private & $61(25.1)$ & $98(40.3)$ & 45 (18.5) & $39(16.0)$ & & $213(87.7)$ & $22(9.1)$ & $8(3.3)$ & & $91(37.4)$ & $86(35.4)$ & $66(27.2)$ & \\
\hline \multicolumn{14}{|c|}{$\begin{array}{l}\text { Repeated school } \\
\text { year }\end{array}$} \\
\hline No & $117(16.6)$ & $265(37.6)$ & $162(23.0)$ & $161(22.8)$ & 0.010 & 581 (82.4) & $86(12.2)$ & $38(5.4)$ & 0.304 & $161(22.8)$ & 281 (39.9) & $263(37.3)$ & 0.021 \\
\hline Yes & $22(12.4)$ & 49 (27.7) & $54(30.5)$ & 52 (29.4) & & 141 (79.7) & 21 (11.9) & $15(8.5)$ & & $25(14.1)$ & $71(40.1)$ & $81(45.8)$ & \\
\hline \multicolumn{14}{|c|}{$\begin{array}{l}\text { Sleep duration } \\
\text { (hours/day) }\end{array}$} \\
\hline$\leq 8$ & $16(13.6)$ & 39 (33.1) & $28(23.7)$ & 35 (29.7) & 0.620 & 93 (78.8) & $19(16.1)$ & $6(5.1)$ & 0.679 & $23(19.5)$ & $42(35.6)$ & $53(44.9)$ & 0.663 \\
\hline$>8 \leq 9$ & 74 (17.7) & $145(34.6)$ & $105(25.1)$ & 95 (22.7) & & $344(82.1)$ & $50(11.9)$ & $25(6.0)$ & & $87(20.8)$ & $173(41.3)$ & 159 (37.9) & \\
\hline$>9$ & $50(14.5)$ & $130(37.7)$ & $82(23.8)$ & $83(24.1)$ & & $285(82.6)$ & 38 (11.0) & $22(6.4)$ & & 77 (22.3) & $136(39.4)$ & $132(38.3)$ & \\
\hline \multicolumn{14}{|l|}{ Ever smoke } \\
\hline No & 98 (16.0) & $222(36.3)$ & $156(25.5)$ & $135(25.5)$ & 0.199 & $509(83.3)$ & $69(11.3)$ & $33(5.4)$ & 0.483 & $136(22.3)$ & $252(41.2)$ & $223(36.5)$ & 0.055 \\
\hline Yes & $29(13.1)$ & 79 (35.6) & $50(22.5)$ & $64(28.8)$ & & 177 (79.7) & $31(14.0)$ & $14(6.3)$ & & $36(16.2)$ & $87(39.2)$ & $99(44.6)$ & \\
\hline \multicolumn{14}{|c|}{ Physical activity } \\
\hline$<1 /$ week & $65(14.1)$ & 149 (32.3) & $119(25.8)$ & $129(27.9)$ & 0.004 & $383(82.9)$ & $53(11.5)$ & $26(5.6)$ & 0.953 & $85(18.4)$ & $185(40.0)$ & $192(41.6)$ & 0.051 \\
\hline$\geq 1 /$ week & $64(17.1)$ & $153(40.9)$ & $87(23.3)$ & 70 (18.7) & & $307(82.1)$ & 45 (12.0) & $22(5.9)$ & & $90(24.1)$ & $155(41.4)$ & $129(34.5)$ & \\
\hline \multicolumn{14}{|c|}{$\mathrm{BMI}{ }^{*}\left(\mathrm{~kg} / \mathrm{m}^{2}\right)$} \\
\hline$<85^{\text {th }}$ & $43(10.1)$ & $140(32.8)$ & $115(26.9)$ & $129(30.2)$ & 0.218 & 341 (79.9) & $56(13.1)$ & $30(7.0)$ & 0.684 & $67(15.7)$ & $162(37.9)$ & $198(46.4)$ & 0.446 \\
\hline$\geq 85^{\text {th }}$ & $12(7.6)$ & $57(36.1)$ & $52(32.9)$ & 37 (23.4) & & $128(81.0)$ & $22(13.9)$ & $8(5.1)$ & & $28(17.7)$ & $66(41.8)$ & $64(40.5)$ & \\
\hline $\begin{array}{l}\text { Depressive } \\
\text { symptomato } \\
\text { |** }\end{array}$ & \multicolumn{13}{|c|}{ 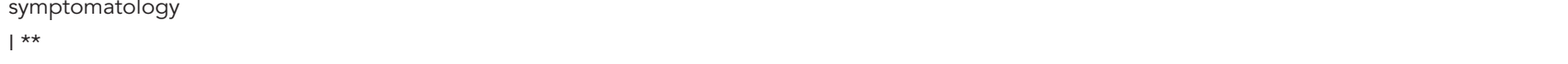 } \\
\hline$<13$ & $103(15.0)$ & $253(36.9)$ & $167(24.4)$ & $162(23.6)$ & 0.575 & $576(84.1)$ & $73(10.7)$ & $36(5.3)$ & 0.031 & $143(20.9)$ & $286(41.8)$ & $256(37.4)$ & 0.314 \\
\hline$\geq 13$ & $28(18.5)$ & $48(31.8)$ & $39(25.8)$ & $36(23.8)$ & & $114(75.5)$ & 27 (17.9) & $10(6.6)$ & & $34(22.5)$ & $53(35.1)$ & $64(42.4)$ & \\
\hline
\end{tabular}

* Body mass index according to US Centers for Disease Control and Prevention percentiles (Kuczmarski et al. 20);

** Non-depressed if score $<13$ and depressed if score $\geq 13$ depressive symptoms on Beck Depression Inventory, second edition. 
Daily mean of television, computer and total media use, according to parents' and adolescents' characteristics, in boys.

\begin{tabular}{|c|c|c|c|c|c|c|c|c|c|c|c|c|c|}
\hline & \multicolumn{5}{|c|}{ Television (hours/day) } & \multicolumn{4}{|c|}{ Computer games (hours/day) } & \multicolumn{4}{|c|}{ Total media (hours/day) } \\
\hline & $\begin{array}{c}\leq 1 \\
n(\%)\end{array}$ & $\begin{array}{c}>1 \leq 2 \\
n(\%)\end{array}$ & $\begin{array}{c}>2 \leq 3 \\
n(\%)\end{array}$ & $\begin{array}{c}>3 \\
n(\%)\end{array}$ & p-value & $\begin{array}{c}\leq 1 \\
\mathrm{n}(\%)\end{array}$ & $\begin{array}{c}>1 \leq 2 \\
n(\%)\end{array}$ & $\begin{array}{c}>2 \\
n(\%)\end{array}$ & $\mathrm{p}$-value & $\begin{array}{c}\leq 2 \\
n(\%)\end{array}$ & $\begin{array}{c}>2 \leq 3 \\
n(\%)\end{array}$ & $\begin{array}{c}>3 \\
n(\%)\end{array}$ & p-value \\
\hline \multicolumn{14}{|c|}{$\begin{array}{l}\text { Living with both } \\
\text { parents }\end{array}$} \\
\hline No & $16(11.3)$ & $45(31.7)$ & $34(23.9)$ & $47(33.1)$ & 0.016 & $63(44.4)$ & $42(29.6)$ & $37(26.1)$ & 0.007 & $10(7.0)$ & $47(33.1)$ & 85 (59.9) & 0.073 \\
\hline Yes & $108(17.8)$ & $221(36.3)$ & $149(24.5)$ & $130(21.4)$ & & $329(54.1)$ & $186(30.6)$ & $93(15.3)$ & & $61(10.0)$ & $247(40.6)$ & $300(49.3)$ & \\
\hline \multicolumn{14}{|c|}{ Parents' age (years) } \\
\hline$\leq 40$ & $7(6.5)$ & $36(33.3)$ & $33(30.6)$ & $32(29.6)$ & 0.014 & $50(46.3)$ & 37 (34.3) & $21(19.4)$ & 0.156 & $5(4.6)$ & $32(29.6)$ & $71(65.7)$ & 0.001 \\
\hline $41-50$ & $84(20.4)$ & $148(36.0)$ & $89(21.7)$ & $90(21.9)$ & & $229(55.7)$ & $119(29.0)$ & $63(15.3)$ & & $50(12.2)$ & $174(42.3)$ & $187(45.5)$ & \\
\hline$\geq 51$ & $25(15.1)$ & $56(33.7)$ & $42(25.3)$ & $43(25.9)$ & & $79(47.6)$ & $50(30.1)$ & $37(22.3)$ & & $10(6.0)$ & $65(39.2)$ & $91(54.8)$ & \\
\hline \multicolumn{14}{|c|}{$\begin{array}{l}\text { Parental education } \\
\text { (years) }\end{array}$} \\
\hline$\leq 6$ & $15(7.9)$ & $53(27.9)$ & $62(32.6)$ & $60(31.6)$ & $<0.001$ & $144(78.7)$ & $22(12.0)$ & $17(9.3)$ & 0.004 & $6(3.4)$ & 55 (30.9) & $117(65.7)$ & $<0.001$ \\
\hline $7-12$ & $48(12.6)$ & $137(36.1)$ & $93(24.5)$ & $102(26.8)$ & & $308(79.6)$ & $36(9.3)$ & $43(11.1)$ & & $34(9.1)$ & $142(38.2)$ & $196(52.7)$ & \\
\hline$>12$ & $68(31.5)$ & $84(38.9)$ & $40(18.5)$ & $24(11.1)$ & & $195(89.0)$ & $18(8.2)$ & $6(2.7)$ & & 35 (16.4) & 105 (49.1) & $74(34.6)$ & \\
\hline \multicolumn{14}{|c|}{ Type of school } \\
\hline Public & $80(13.3)$ & $198(33.0)$ & $161(26.0)$ & $161(26.0)$ & $<0.001$ & $302(50.3)$ & $181(30.2)$ & $117(19.5)$ & 0.012 & $45(7.5)$ & $223(37.2)$ & $332(55.3)$ & $<0.001$ \\
\hline Private & $54(40.3)$ & $82(29.3)$ & $33(16.8)$ & $27(13.8)$ & & $116(59.2)$ & $59(30.1)$ & $21(10.7)$ & & $33(16.8)$ & $93(47.4)$ & 70 (35.7) & \\
\hline \multicolumn{14}{|c|}{ Repeated school } \\
\hline \multicolumn{14}{|l|}{ year } \\
\hline No & $114(19.0)$ & $217(36.2)$ & $136(22.7)$ & $133(22.2)$ & 0.004 & $321(53.5)$ & $182(30.3)$ & $97(16.2)$ & 0.339 & $67(11.2)$ & $243(40.5)$ & $290(48.3)$ & 0.010 \\
\hline Yes & $18(9.5)$ & $62(22.2)$ & $57(29.5)$ & $53(28.5)$ & & $93(48.9)$ & $58(30.5)$ & 39 (20.5) & & $9(4.7)$ & $71(37.4)$ & $110(57.9)$ & \\
\hline \multicolumn{14}{|c|}{ Sleep duration } \\
\hline \multicolumn{14}{|l|}{ (h/day) } \\
\hline$\leq 8$ & $9(11.5)$ & $19(22.4)$ & $23(29.5)$ & $27(34.6)$ & 0.020 & $33(42.3)$ & 27 (34.6) & $18(23.1)$ & 0.189 & $4(5.1)$ & $24(30.8)$ & $50(64.1)$ & 0.011 \\
\hline$>8 \leq 9$ & $58(15.8)$ & $129(35.1)$ & $85(23.2)$ & $95(34.6)$ & & $187(51.0)$ & $114(31.1)$ & $66(18.0)$ & & $37(10.1)$ & $134(36.5)$ & $196(53.4)$ & \\
\hline$>9$ & $67(19.1)$ & $132(37.6)$ & $86(24.5)$ & $66(18.8)$ & & $198(56.4)$ & 99 (28.2) & $54(15.4)$ & & $37(10.5)$ & $158(45.0)$ & $156(44.4)$ & \\
\hline \multicolumn{14}{|l|}{ Ever smoke } \\
\hline No & $104(17.5)$ & $215(36.2)$ & $145(24.4)$ & $130(21.9)$ & 0.164 & $320(53.9)$ & $179(30.1)$ & $95(16.0)$ & 0.094 & $60(10.1)$ & $240(40.4$ & $294(49.5)$ & 0.102 \\
\hline Yes & $20(14.9)$ & $39(29.1)$ & $35(26.1)$ & $40(29.9)$ & & $61(45.5)$ & $42(31.3)$ & $31(23.1)$ & & $11(8.2)$ & $43(32.1)$ & $80(59.7)$ & \\
\hline \multicolumn{14}{|c|}{ Physical activity } \\
\hline$<1 /$ week & $34(17.3)$ & $71(36.2)$ & $44(22.4)$ & $47(24.0)$ & 0.916 & $99(50.5)$ & $66(33.7)$ & $31(15.8)$ & 0.386 & $19(9.7)$ & 75 (38.3) & $102(52.0)$ & 0.928 \\
\hline$\geq 1 /$ week & $90(16.7)$ & $189(35.0)$ & $135(25.0)$ & $126(23.3)$ & & $289(53.5)$ & $154(28.5)$ & $97(18.0)$ & & $52(9.6)$ & $215(39.8)$ & $273(50.6)$ & \\
\hline \multicolumn{14}{|l|}{$\mathrm{BMI} *\left(\mathrm{~kg} / \mathrm{m}^{2}\right)$} \\
\hline$<85^{\text {th }}$ & $43(11.2)$ & $130(33.8)$ & $99(25.7)$ & $113(29.4)$ & 0.643 & $204(53.0)$ & $105(27.3)$ & $76(19.7)$ & 0.443 & $24(6.2)$ & $141(36.6)$ & 220 & 0.909 \\
\hline$\geq 85^{\text {th }}$ & $12(8.8)$ & $43(31.6)$ & $42(30.9)$ & $39(28.7)$ & & $70(51.5)$ & $44(32.4)$ & $22(16.2)$ & & $9(6.6)$ & $47(34.6)$ & $80(58.8)$ & \\
\hline \multicolumn{14}{|l|}{ Depressive } \\
\hline \multicolumn{14}{|c|}{ symptomatology I ** } \\
\hline$<13$ & $114(16.5)$ & $248(36.0)$ & $168(24.4)$ & $159(23.1)$ & 0.890 & $360(52.2)$ & $216(31.3)$ & $113(16.4)$ & 0.107 & $67(9.7)$ & 275 (39.9) & $347(50.4)$ & 0.119 \\
\hline$\geq 13$ & $8(18.2)$ & $14(31.8)$ & $10(22.7)$ & $12(27.3)$ & & $23(52.3)$ & $9(20.5)$ & $12(27.3)$ & & $2(4.5)$ & $13(29.5)$ & $29(65.9)$ & \\
\hline
\end{tabular}

* Body mass index according to US Centers for Disease Control and Prevention percentiles (Kuczmarski et al. 20);

** Non-depressed if score $<13$ and depressed if score $\geq 13$ depressive symptoms on Beck Depression Inventory, second edition.

once a week (proportional OR $=0.73$; 95\%CI: 0.56-0.97). Regarding computer exposure, girls from private schools were also less likely to use the computer $(\mathrm{OR}=0.55$; 95\%CI: 0.35-0.85). In contrast, those who were depressed had greater odds of using the computer for longer $(\mathrm{OR}=1.71$ 95\%CI: 1.11-2.61). Considering the overall media exposure, the odds of higher exposure was lower in those from private schools (proportional OR = 0.53; 95\%CI: 0.39-0.71) and higher in ever smok- 
ers $($ proportional OR $=1.42 ; 95 \% \mathrm{CI}: 1.06-1.91)$ (Table 4).

Regarding boys, there was a higher probability of watching television more time, among those who did not live with both parents (proportional $\mathrm{OR}=1.61$; 95\%CI: 1.13-2.28) and among those who ever smoked (proportional OR $=1.49 ; 95 \% \mathrm{CI}$ : 1.01-2.08). Those attending private schools (proportional OR $=0.54$; 95\%CI: $0.37-0.77$ ) and those who sleep more than 9 hours (proportional OR = 0.48; 95\%CI: $0.30-0.78$ ) were less likely to spend time watching television. Similar associations were found for computer use and the overall media (Table 5).

\section{Discussion}

In the present study we show a high prevalence of adolescents using media for more than 2 hours per day, the maximum time period recommended for daily media use 21 , which is consistent with other studies $22,23,24$. In line with previous data, adolescents reported spending more time watching television than using the computer 4,24 . At the weekend the use is considerably higher than at weekdays. This may have relevant implications for the planning of the measurement of media use, given that if the amount of time is questioned as a median time (without making any distinction between week and weekend days), the media use time may be underestimated, since adolescents may be referring to their use during weekdays, that are more frequent.

In accordance with other studies, we found no gender differences in the time spent watching television $4,24,25$. This notwithstanding, gender may be a differentiating factor in media use. Boys are significantly more likely to use a computer than girls, resulting in a higher time using media $16,24,25$. This gender difference may be explained by the fact that computer use in the present study includes playing games, which is an activity preferred by boys, while girls use computers more for social activities, such as sending e-mails and instant messaging 1.

Generally, adolescents that belong to a less favoured social class presented higher levels of media use 1,16,26,27. A similar pattern was found among adolescents with younger and older parents, as well as those that did not live with both parents. Higher levels of parental education and household income seem to be associated with the existence of rules governing any kind of media behaviour ${ }^{1}$. A social environment with more permissive rules, including the absence of clear rules and setting limits on screen time may be a possible explanation for our results regarding parents' characteristics 28 . On the other hand, adolescents belonging to higher social classes may have more opportunities to invest their time on extra-curricular activities, namely practicing sports.

Our study illustrates that longer television and computer exposure was associated with unhealthy behaviours, including tobacco use, less sleep time and sedentary behaviours, in line with previous findings 29 . These findings are consistent with the idea that adolescents who engage in a particular unhealthy behaviour may be more prone to engage in other unhealthy behaviours 30,31 . Although it was not evaluated in this study, evidence shows that individuals with a greater tendency for thrill seeking are more likely to consume television and computer games. These individuals are also more prone to engage in activities that could endanger their well-being, such as cigarette smoking, given their disposition to search for intense experiences 1 .

We also verified that girls that use computers more frequently presented more depressive symptomatology. This finding was reported by Primack et al. 32 particularly in boys, though not in girls. The lack of association for boys in our study may be due to a low statistical power, given that boys have less depressive symptomatology than girls.

We also found an association between time watching television and repeating a school year. This association may be explained by the displacement of study activities for time spent watching television, leading to a more negative school performance 10,33 . However due to the cross-sectional nature of our study, it is still unclear if this media exposure may be the cause or the consequence of school retention. We cannot also exclude the hypothesis that this may just reflect the socioeconomic environment, which predisposes a worse performance at school.

Notwithstanding the aforementioned results, this raises questions about the role of educational institutions on adolescents' development. It has been shown that schools represent a resource to promote adolescents' health. It has also been shown that school achievement is positively linked with lifelong health outcomes 34 . A particular challenge for schools is helping adolescents to acquire knowledge and skills about media potential to influence them (positive and negatively) and society. According to our results, those who are at risk of greater media use may be those more economically and socially disadvantaged. In this line of thought, schools should have the role of building resilience and ensuring a curriculum that allows all adolescents to 
Associations between adolescents' characteristics and global daily mean of television, computer and total media use, in girls.

\begin{tabular}{|c|c|c|c|c|c|c|}
\hline & \multicolumn{2}{|c|}{ Daily mean of television use * } & \multicolumn{2}{|c|}{ Daily mean of computer use * } & \multicolumn{2}{|c|}{ Daily mean of total media use * } \\
\hline & $\begin{array}{c}\text { Crude } \\
\text { proportional } \\
\text { OR }(95 \% \mathrm{Cl})\end{array}$ & $\begin{array}{c}\text { Adjusted } \\
\text { proportional } \\
\text { OR }(95 \% \mathrm{Cl})\end{array}$ & $\begin{array}{l}\text { Crude OR } \\
(95 \% \mathrm{Cl})\end{array}$ & $\begin{array}{l}\text { Adjusted OR } \\
\qquad(95 \% \mathrm{Cl})\end{array}$ & $\begin{array}{c}\text { Crude } \\
\text { proportional } \\
\text { OR }(95 \% \mathrm{Cl})\end{array}$ & $\begin{array}{c}\text { Adjusted } \\
\text { proportional } \\
\text { OR }(95 \% \mathrm{Cl})\end{array}$ \\
\hline \multicolumn{7}{|c|}{ Living with both parents } \\
\hline Yes & 1.00 & 1.00 & 1.00 & 1.00 & 1.00 & 1.00 \\
\hline No & $1.21(0.90-1.63)$ & $1.13(0.84-1.54)$ & $0.77(0.50-1.18)$ & $0.76(0.49-1.18)$ & $1.20(0.89-1.61)$ & $1.12(0.83-1.51)$ \\
\hline \multicolumn{7}{|c|}{ Parents' age (years) } \\
\hline$\leq 40$ & 1.00 & 1.00 & 1.00 & 1.00 & 1.00 & 1.00 \\
\hline $41-50$ & $0.79(0.55-1.14)$ & $1.06(0.72-1.57)$ & $0.75(0.46-1.22)$ & $0.81(0.48-1.34)$ & $0.69(0.47-0.99)$ & $0.88(0.60-1.30)$ \\
\hline$\geq 51$ & $0.94(0.61-1.44)$ & $1.15(0.73-1.80)$ & $0.58(0.32-1.06)$ & $0.62(0.33-1.14)$ & $0.76(0.50-0.86)$ & $0.900 .58-1.40)$ \\
\hline \multicolumn{7}{|c|}{ Type of school } \\
\hline Public & 1.00 & 1.00 & 1.00 & 1.00 & 1.00 & 1.00 \\
\hline Private & $0.47(0.35-0.63)$ & $0.59(0.43-0.81)$ & $0.55(0.36-0.85)$ & $0.55(0.35-0.85)$ & $0.43(0.33-0.58)$ & $0.53(0.39-0.71)$ \\
\hline \multicolumn{7}{|c|}{ Repeated school year } \\
\hline No & 1.00 & 1.00 & 1.00 & 1.00 & 1.00 & 1.00 \\
\hline Yes & $1.61(1.19-2.19)$ & $1.16(0.83-1.62)$ & $0.84(0.55-1.27)$ & $0.85(0.55-1.31)$ & $1.57(1.15-2.14)$ & $1.15(0.82-1.61)$ \\
\hline \multicolumn{7}{|c|}{ Sleep duration (hours/day) } \\
\hline$\leq 8$ & 1.00 & 1.00 & 1.00 & 1.00 & 1.00 & 1.00 \\
\hline$>8 \leq 9$ & $0.76(0.52-1.11)$ & $0.78(0.52-1.16)$ & $0.81(0.49-1.35)$ & $0.80(0.48-1.34)$ & $0.80(0.55-1.16)$ & $0.78(0.53-1.16)$ \\
\hline$>9$ & $0.78(0.53-1.15)$ & $0.73(0.49-1.10)$ & $0.78(0.47-1.32)$ & $0.76(0.45-1.28)$ & $0.82(0.56-1.21)$ & $0.76(0.51-1.14)$ \\
\hline \multicolumn{7}{|l|}{ Ever smoke } \\
\hline No & 1.00 & 1.00 & 1.00 & 1.00 & 1.00 & 1.00 \\
\hline Yes & $1.25(0.93-1.67)$ & $1.26(0.94-1.70)$ & $1.27(0.86-1.87)$ & $1.27(0.86-1.87)$ & $1.39(1.04-1.85)$ & $1.42(1.06-1.91)$ \\
\hline \multicolumn{7}{|c|}{ Frequency of physical activity } \\
\hline$<1 /$ week & 1.00 & 1.00 & 1.00 & 1.00 & 1.00 & 1.00 \\
\hline$\geq 1 /$ week & $0.62(0.47-0.80)$ & $0.73(0.56-0.97)$ & $1.06(0.74-1.51)$ & $1.11(0.77-1.60)$ & $0.76(0.59-0.98)$ & $0.87(0.67-1.14)$ \\
\hline \multicolumn{7}{|c|}{$\mathrm{BMI} * \star\left(\mathrm{kg} / \mathrm{m}^{2}\right)$} \\
\hline$<85^{\text {th }}$ & 1.00 & 1.00 & 1.00 & 1.00 & 1.00 & 1.00 \\
\hline$\geq 85^{\text {th }}$ & $0.86(0.62-1.21)$ & $0.91(0.64-1.28)$ & $0.93(0.59-1.48)$ & $0.94(0.59-1.50)$ & $0.90(0.64-1.27)$ & $0.94(0.66-1.33)$ \\
\hline \multicolumn{7}{|c|}{ 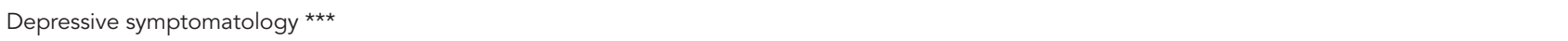 } \\
\hline$<13$ & - & - & 1.00 & 1.00 & - & - \\
\hline$\geq 13$ & - & - & $1.72(1.12-2.62)$ & $1.71(1.11-2.61)$ & - & - \\
\hline
\end{tabular}

95\% Cl: 95\% confidence interval; OR: odds ratio.

* Television use categorized into $\leq 1$ hour; $>1 \leq 2$ hours and $>2$ hours; computer use categorized into two categories, $\leq 2$ hours and $>2$ hours; and total media use categorized into $\leq 2$ hours; $>2 \leq 3$ hours and $>3$ hours;

** Body mass index according to US Centers for Disease Control and Prevention percentiles (Kuczmarski et al. 20);

$\star \star \star$ Non-depressed if score $<13$ and depressed if score $\geq 13$ depressive symptoms on Beck Depression Inventory, second edition.

achieve the best physical and behavioural development 21,34,35.

With regard limitations, we reported that questionnaires were filled out by adolescents and by their parents at home, which may have caused an underestimation of the amount of media time used, due to what is socially desirable. On the other hand, we only measured the quantity of media exposure and not its content, which could have provided additional data that goes beyond the scope of this study. Another important limitation was related with the cross-sectional nature of our study which prevents the identification of the temporal relation between some associations, for instance, between media exposure time and sports activities. One final limitation is the number of adolescents excluded from the study. However, this is not thought to compromise the results, because, in general, there were no differences between included and excluded 
Associations between adolescents' characteristics and global daily mean of television, computer and total media use, in boys

\begin{tabular}{|c|c|c|c|c|c|c|}
\hline & \multicolumn{2}{|c|}{ Daily mean of television use * } & \multicolumn{2}{|c|}{ Daily mean of computer use * } & \multicolumn{2}{|c|}{ Daily mean of total media use * } \\
\hline & $\begin{array}{c}\text { Crude } \\
\text { proportional OR } \\
(95 \% \mathrm{Cl})\end{array}$ & $\begin{array}{c}\text { Adjusted } \\
\text { proportional OR } \\
(95 \% \mathrm{Cl})\end{array}$ & $\begin{array}{c}\text { Crude OR } \\
(95 \% \mathrm{Cl})\end{array}$ & $\begin{array}{l}\text { Adjusted OR } \\
(95 \% \mathrm{Cl})\end{array}$ & $\begin{array}{c}\text { Crude } \\
\text { proportional OR } \\
(95 \% \mathrm{Cl})\end{array}$ & $\begin{array}{c}\text { Adjusted } \\
\text { proportional OR } \\
(95 \% \mathrm{Cl})\end{array}$ \\
\hline \multicolumn{7}{|c|}{ Living with both parents } \\
\hline Yes & 1.00 & 1.00 & 1.00 & 1.00 & 1.00 & 1.00 \\
\hline No & $1.66(1.18-2.34)$ & $1.61(1.13-2.28)$ & $1.48(1.02-2.14)$ & $1.46(1.01-2.11)$ & $1.45(1.02-2.07)$ & $1.38(0.96-1.99)$ \\
\hline \multicolumn{7}{|c|}{ Parents' age (years) } \\
\hline$\leq 40$ & 1.00 & 1.00 & 1.00 & 1.00 & 1.00 & 1.00 \\
\hline $41-50$ & $0.57(0.38-0.84)$ & $0.75(0.49-1.13)$ & $0.69(0.45-1.05)$ & $0.76(0.49-1.19)$ & $0.43(0.28-0.66)$ & $0.56(0.36-0.88)$ \\
\hline$\geq 51$ & $0.75(0.48-1.18)$ & $0.89(0.55-1.42)$ & $0.95(0.58-1.54)$ & $1.01(0.62-1.67)$ & $0.64(0.39-1.04)$ & $0.75(0.45-1.26)$ \\
\hline \multicolumn{7}{|c|}{ Type of school } \\
\hline Public & 1.00 & 1.00 & 1.00 & 1.00 & 1.00 & 1.00 \\
\hline Private & $0.39(0.28-0.55)$ & $0.54(0.37-0.77)$ & $0.70(0.50-0.97)$ & $0.72(0.52-1.01)$ & $0.45(0.33-0.61)$ & $0.61(0.44-0.84)$ \\
\hline \multicolumn{7}{|c|}{ Repeated school year } \\
\hline No & 1.00 & 1.00 & 1.00 & 1.00 & 1.00 & 1.00 \\
\hline Yes & $1.56(1.15-2.12)$ & $0.90(0.64-1.26)$ & $0.83(0.60-1.16)$ & $0.88(0.63-1.24)$ & $1.49(1.09-2.05)$ & $1.05(0.75-1.49)$ \\
\hline \multicolumn{7}{|c|}{ Sleep duration (hours/day) } \\
\hline$\leq 8$ & 1.00 & 1.00 & 1.00 & 1.00 & 1.00 & 1.00 \\
\hline$>8 \leq 9$ & $0.59(0.37-0.92)$ & $0.74(0.46-1.19)$ & $0.71(0.43-1.16)$ & $0.69(0.42-1.14)$ & $0.62(0.38-1.02)$ & $0.78(0.47-1.29)$ \\
\hline$>9$ & $0.44(0.28-0.70)$ & $0.48(0.30-0.78)$ & $0.57(0.35-0.93)$ & $0.55(0.33-0.91)$ & $0.46(0.28-0.75)$ & $0.53(0.32-0.89)$ \\
\hline \multicolumn{7}{|l|}{ Ever smoke } \\
\hline No & 1.00 & 1.00 & 1.00 & 1.00 & 1.00 & 1.00 \\
\hline Yes & $1.49(1.05-2.12)$ & $1.45(1.01-2.08)$ & $1.40(0.96-2.04)$ & $1.44(0.98-2.11)$ & $1.49(1.04-2.16)$ & $1.49(1.02-2.20)$ \\
\hline \multicolumn{7}{|c|}{ Frequency of physical activity } \\
\hline$<1 /$ week & 1.00 & 1.00 & 1.00 & 1.00 & 1.00 & 1.00 \\
\hline$\geq 1 /$ week & $1.04(0.76-1.42)$ & $1.30(0.94-1.81)$ & $0.89(0.64-1.23)$ & $0.92(0.66-1.28)$ & $1.00(0.73-1.36)$ & $1.23(0.86-1.70)$ \\
\hline \multicolumn{7}{|c|}{$\mathrm{BMI}{ }^{* \star}\left(\mathrm{kg} / \mathrm{m}^{2}\right)$} \\
\hline$<85^{\text {th }}$ & 1.00 & 1.00 & 1.00 & 1.00 & 1.00 & 1.00 \\
\hline$\geq 85^{\text {th }}$ & $1.10(0.77-1.58)$ & $1.14(0.79-1.66)$ & $1.06(0.72-1.57)$ & $1.07(0.72-1.58)$ & $1.03(0.70-1.50)$ & $1.06(0.72-1.58)$ \\
\hline \multicolumn{7}{|c|}{ 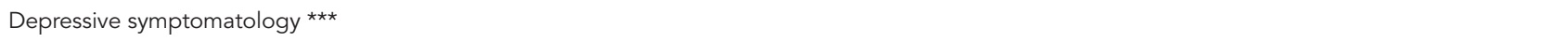 } \\
\hline$<13$ & 1.00 & 1.00 & 1.00 & 1.00 & 1.00 & 1.00 \\
\hline$\geq 13$ & $1.15(0.65-2.05)$ & $1.17(0.64-2.14)$ & $1.00(0.54-1.84)$ & $1.02(0.55-1.89)$ & $1.71(0.92-3.16)$ & $1.56(0.82-2.97)$ \\
\hline
\end{tabular}

95\% Cl: 95\% confidence interval; OR: odds ratio.

* Television use categorized into $\leq 1$ hour; $>1 \leq 2$ hours and $>2$ hours; computer use categorized into two categories, $\leq 2$ hours and $>2$ hours; and total media use categorized into $\leq 2$ hours; $>2 \leq 3$ hours and $>3$ hours;

** Body mass index according to US Centers for Disease Control and Prevention percentiles (Kuczmarski et al. 20);

*** Non-depressed if score $<13$ and depressed if score $\geq 13$ depressive symptoms on Beck Depression Inventory, second edition.

participants regarding the proportion of individual and behavioural characteristics (including body mass index, absence of chronic illness, smoking or practicing exercise). So, it seems reasonable to expect that the distribution of other behaviours should be similar, such as media use and associated characteristics. Among those excluded we had a higher proportion of boys, this may be due to the fact that boys are more prone to not complete the questionnaires entirely when compared with girls, who may be more oriented to details and perfection, nevertheless, as our results were presented by gender it is not expected to be affected by this bias. The higher proportion of adolescents with a lower socioeconomic status that were excluded could represent a selection bias. Moreover, as we are able to identify the effect of socioeconomic status as a determinant of media exposure, we could expect that if those adolescents were included in the analysis, its im- 
pact could be in the direction of strengthening the results already presented. However, we must admit that the estimates of the prevalence of use of television are probably under-estimated. Despite these limitations, the study has the benefit of differentiating media use between weekdays and weekends, which is important, because its determinants may differ considerably, requiring different forms of consideration and intervention. On the other hand, it is also worth noting that our study considers only 13 -year-old adolescents, from a representative sample of students at this age, enabling a more homogeneous group, at a stage of their life-cycle that is marked by constant change and transition. Furthermore, since school attendance is compulsory at this age in Portugal our sample is representative of adolescents at this age.

\section{Conclusion}

More than half of 13-year-old adolescents reported using the television and computer more than the recommended 2 hours per day with a higher exposure in boys, especially on weekdays. Besides the association with unhealthier be- haviours, we also found higher levels of media use among socially disadvantaged adolescents (lower social class and less structured families), which may increase their exposure to a negative influence of the media.

In the context of public health, findings from this study suggest that an intervention conceptualized to reduce time watching television and using the computer should include specific strategies oriented to the definition of alternative occupational activities to occupy adolescents' free time, especially during the weekend, and which should be adjusted to families with a lower socioeconomical status.

Since a drastic reduction in media time use is not expected, the intervention should equally target the capacity of parents to adequately choose the content of media to which their children are exposed. Concomitantly, it is important to promote adolescents' resilience, in order to contribute to their own capacitation on discriminating prejudicial patterns of media use and help them to identify and implement measures to balance this behaviour with other healthy and stimulating activities on their free time, given they are in a period of development and consolidation of their identities.

\section{Resumen}

El objetivo del estudio fue identificar las características socioeconómicas y de comportamiento asociadas con el uso de medios de comunicación en la adolescencia temprana. La muestra se constituyó por 1.680 adolescentes (884 niñas y 796 niños) de 13 años de escuelas públicas y privadas en Porto, Portugal, en 2003/2004. Estos completaron cuestionarios que contenían características sociodemográficas y de comportamiento, incluyendo el tiempo empleado en ver la televisión y el ordenador, en días de semana y fines de semana. Regresiones logísticas ordinales y proporcionales mostraron que los adolescentes de las escuelas públicas, los que dormían menos, consumían tabaco y practican poca actividad física eran más propensos a usar más medios de comunicación. Los niños que viven con un solo padre o con padres más jóvenes o más viejos los utilizaban también más. Así, además de la asociación con comportamientos poco saludables, se concluyó que un mayor uso de los medios de comunicación en los adolescentes de familias de clase social baja y de familias menos estructuradas, lo que puede aumentar su permeabilidad a la influencia negativa de estos medios.

Medios de Comunicación; Adolescente; Conducta del Adolescente; Televisión 


\section{Contributors}

J. Godinho drafted the first version of the manuscript performed the statistical analyses and contributed to the interpretation of the results. J. Araújo contributed to the interpretation of the results and critically reviewed the manuscript. H. Barros coordinated the data collection and critically reviewed the manuscript. E. Ramos conceived and designed the study, coordinated and supervised the data collection and contributed to the interpretation and discussion of the data.

\section{References}

1. Rideout V, Roberts D, Foehr U. Generation M: me dia in the lives of 8-18 year-olds. Sand Hill Road: The Henry J. Kaiser Family Foundation; 2005.

2. Brown JD, Witherspoon EM. The mass media and American adolescents' health. J Adolesc Health 2002; 31(6 Suppl):153-70.

3. Villani S. Impact of media on children and adolescents: a 10-year review of the research. J Am Acad Child Adolesc Psychiatry 2001; 40:392-401.

4. Kappos AD. The impact of electronic media on mental and somatic children's health. Int J Hyg Environ Health 2007; 210:555-62.

5. Subrahmanyam K, Kraut RE, Greenfield PM, Gross EF. The impact of home computer use on children's activities and development. Future Child 2000; 10:123-44.

6. Bar-on ME. The effects of television on child health: implications and recommendations. Arch Dis Child 2000; 83:289-92.

7. Straker L, Pollock C, Maslen B. Principles for the wise use of computers by children. Ergonomics 2009; 52:1386-401.

8. Bickham DS, Rich M. Is television viewing associated with social isolation? Roles of exposure time, viewing context, and violent content. Arch Pediatr Adolesc Med 2006; 160:387-92.

9. Landhuis CE, Poulton R, Welch D, Hancox RJ. Does childhood television viewing lead to attention problems in adolescence? Results from a prospective longitudinal study. Pediatrics 2007; 120:532-7.

\section{Acknowledgement}

The study was supported by grants from the Portuguese Foundation for Science and Technology: POCTI/ SAU-ESP/62399/2004, FCOMP-01-0124-FEDER-015750 (Ref. FCT PTDC/SAU-EPI/115254/2009), and SFRH/ $\mathrm{BD} / 78153 / 2011$ to JA.
10. Sharif I, Wills TA, Sargent JD. Effect of visual media use on school performance: a prospective study. J Adolesc Health 2010; 46:52-61.

11. Thomee S, Dellve L, Harenstam A, Hagberg M. Perceived connections between information and communication technology use and mental symptoms among young adults - a qualitative study. BMC Public Health 2010; 10:66.

12. Zimmerman FJ, Christakis DA. Children's television viewing and cognitive outcomes: a longitudinal analysis of national data. Arch Pediatr Adolesc Med 2005; 159:619-25.

13. Zimmerman FJ, Christakis DA. Associations between content types of early media exposure and subsequent attentional problems. Pediatrics 2007; 120:986-92.

14. Dworak M, Schierl T, Bruns T, Struder HK. Impact of singular excessive computer game and television exposure on sleep patterns and memory performance of school-aged children. Pediatrics 2007; 120:978-85.

15. Johnson JG, Cohen P, Kasen S, First MB, Brook JS. Association between television viewing and sleep problems during adolescence and early adulthood. Arch Pediatr Adolesc Med 2004; 158:562-8.

16. Roe K. Adolescents' media use: a European view. J Adolesc Health 2000; 27(2 Suppl):15-21.

17. Ramos E, Barros H. Family and school determinants of overweight in 13-year-old Portuguese adolescents. Acta Paediatr 2007; 96:281-6. 
18. Beck AT, Steer RA, Brown GK. Manual for the Beck Depression Inventory-II. San Antonio: Psychological Corporation; 1996.

19. Coelho R, Martins A, Barros H. Clinical profiles relating gender and depressive symptoms among adolescents ascertained by the Beck Depression Inventory II. Eur Psychiatry 2002; 17:222-6.

20. Kuczmarski RJ, Ogden CL, Guo SS, GrummerStrawn LM, Flegal KM, Mei Z, et al. 2000 CDC Growth Charts for the United States: methods and development. Vital Health Stat 11 2002; (246):1-190.

21. American Academy of Pediatrics: Children, adolescents, and television. Pediatrics 2001; 107:423-6.

22. Christakis DA, Ebel BE, Rivara FP, Zimmerman FJ. Television, video, and computer game usage in children under 11 years of age. J Pediatr 2004; 145:652-6.

23. Hancox RJ, Milne BJ, Poulton R. Association between child and adolescent television viewing and adult health: a longitudinal birth cohort study. Lancet 2004; 364:257-62.

24. Marshall SJ, Gorely T, Biddle SJ. A descriptive epidemiology of screen-based media use in youth: a review and critique. J Adolesc 2006; 29:333-49.

25. Rideout V, Roberts D, Foehr U. Generation M2: media in the lives of 8-18 year-olds. Sand Hill Road: The Henry J. Kaiser Family Foundation; 2010.

26. Devis-Devis J, Peiro-Velert C, Beltran-Carrillo VJ, Tomas JM. Screen media time usage of 12-16 yearold Spanish school adolescents: Effects of personal and socioeconomic factors, season and type of day. J Adolesc 2009; 32:213-31.

27. Patriarca A, Di Giuseppe G, Albano L, Marinelli $\mathrm{P}$, Angelillo IF. Use of television, videogames, and computer among children and adolescents in Italy. BMC Public Health 2009; 9:139.
28. Ramirez ER, Norman GJ, Rosenberg DE, Kerr J, Saelens BE, Durant N, et al. Adolescent screen time and rules to limit screen time in the home. J Adolesc Health 2011; 48:379-85.

29. Carson V, Pickett W, Janssen I. Screen time and risk behaviors in 10- to 16-year-old Canadian youth. Prev Med 2011; 52:99-103.

30. Fraga S, Severo M, Costa D, Lopes C, Ramos E. Clustering behaviours among 13-year-old Portuguese adolescents. J Public Health 2011; 19 Suppl 1:S21-7.

31. Jessor RA. Adolescent development and behavioral health. In: Matarazzo J, Weiss S, Herd J, Miller N, Weiss J, editors. Behavioural health: a handbook of health enhancement and disease prevention. New York: Wiley; 1984. p. 69-90.

32. Primack BA, Swanier B, Georgiopoulos AM, Land SR, Fine MJ. Association between media use in adolescence and depression in young adulthood: a longitudinal study. Arch Gen Psychiatry 2009; 66:181-8.

33. Sharif I, Sargent JD. Association between television, movie, and video game exposure and school performance. Pediatrics 2006; 118:e1061-70.

34. Tang KC, Nutbeam D, Aldinger C, St Leger L, Bundy D, Hoffmann AM, et al. Schools for health, edu cation and development: a call for action. Health Promot Int 2009; 24:68-77.

35. Strasburger VC, Jordan AB, Donnerstein E. Health effects of media on children and adolescents. Pediatrics 2010; 125:756-67.

Submitted on 15/May/2013

Final version resubmitted on 12/Aug/2013

Approved on 29/Aug/2013 